\title{
Hopf algebras for ternary algebras
}

\author{
M. Goze* \\ Laboratoire MIA, Université de Haute Alsace, \\ Faculté des Sciences et Techniques, \\ 4 rue des Frères Lumière, \\ 68093 Mulhouse Cedex, France \\ M. Rausch de Traubenberg \\ IPHC-DRS, UdS, CNRS, IN2P3; 23 rue du Loess, 67037 Strasbourg, France
}

(Dated: November 5, 2018)

\begin{abstract}
We construct an universal enveloping algebra associated to the ternary extension of Lie (super)algebras called Lie algebra of order three. A Poincaré-Birkhoff-Witt theorem is proven is this context. It this then shown that this universal enveloping algebra can be endowed with a structure of Hopf algebra. The study of the dual of the universal enveloping algebra enables to define the parameters of the transformation of a Lie algebra of order three. It turns out that these variables are the variables which generate the three-exterior algebra.

PACS numbers: 02.10.Xm, 02.20.Sv, 11.30.Ly
\end{abstract}

\section{INTRODUCTION}

The concept of algebras, that is a vector space $\mathcal{A}$ equipped with a binary product $m_{2}: \mathcal{A} \otimes \mathcal{A} \longrightarrow \mathcal{A}$ is central in physics. Among them Lie algebras and Lie superalgebras are the cornerstone in the construction of models in particles physics. The former lead to a description of space-time and internal symmetries although the latter give rise to supersymmetric extensions of space-time symmetries. The intensive use of Lie (super)algebras is probably due to the central theorems of Coleman \& Mandula [1] and Haag, Lopuszanski \& Sohnius 2] which allow to construct theories based on some specific Lie (super)algebras not contradicting the principles of Quantum Theory and Relativity.

On may naturally wonder whether or not some different algebraic structures should play a role in physics. And in particular one may ask if ternary algebras, that is vector spaces $\mathcal{A}$ equipped with a ternary multiplication: $m_{3}$ : $\mathcal{A} \otimes \mathcal{A} \otimes \mathcal{A} \longrightarrow \mathcal{A}$ should be relevant in the description of some symmetries. Ternary algebras have been considered in physics only occasionally (see for instance [3, 4, 5, 6, 6, 8] and references therein). For some mathematical references one can see $[9,10,11,12]$. Recently they was some revival of interest in ternary algebras when it has been realised

*Electronic address: m.goze@uha.fr

${ }^{\dagger}$ Electronic address: Michel.Rausch@IReS.in2p3.fr 
that a ternary algebra defined by a fully antisymmetric product appears in the description of multiple M2-branes [13]. In [14, 15, 16] an F-ary algebra which can be seen as a possible generalisation of Lie (super)algebras has been considered and named Lie algebra of order $F$. A Lie algebra of order $F$ admits a $\mathbb{Z}_{F}-$ grading $(F=3$ in this paper $)$, the zero-graded part being a Lie algebra. An $F$-fold symmetric product (playing the role of the anticommutator in the case $F=2$ ) expresses the zero graded part in terms of the non-zero graded part. This means that when $F=3$ we have two products: one binary and the second ternary. The first algebras constructed along these lines lead to some (infinite-dimensional) extensions of the Poincaré algebra in $(1+2)$-dimensions and turn out to induce a symmetry which connects relativistics anyons [17]. A major progress in the comprehension of those mathematical structures was undertaken when it was realised that finite dimensional Lie algebras of order $F$ could be defined. Subsequently, a specific (finite-dimensional) Lie algebra or order three, leading to a non-trivial extension of the Poincaré algebra, has been studied together with its implementation in Quantum Field Theory [18, 19, 20, 21]. Then, a general study of the possible non-trivial extensions of the Poincaré algebra in $(1+3)$-dimensions has been undertaken in [16, 22] together with a study of possible kinematical algebras of order three [23].

However, all these mathematical structures have been considered at the level of algebras i.e. at the level of infinitesimal transformations and no groups associated to Lie algebras of order three were considered. At a first glance these two structures seem to be incompatible since for a Lie algebra of order three for some elements only the product of three elements is defined although for a group the product of two elements is always defined. This is rather different to the Lie (super)algebras cases where it is known that Lie (super)groups can be defined. However, on the formal ground it is knows that Lie (super)groups are related to Hopf algebras. Indeed, if $G$ is a simply connected Lie group, there is a duality between $\mathcal{U}(\mathfrak{g})$ (the universal enveloping algebra of $\mathfrak{g}$, the Lie algebra of $G$ ) and $\mathcal{F}(G)($ the vector space of complex valued functions on $G$ ). This means that $\mathcal{F}(G)$ is isomorphic to a subspace of $\mathcal{U}^{*}(\mathfrak{g})$ (the dual of $\mathcal{U}(\mathfrak{g}))[24,[25]$.

The purpose of this paper is to give a first step toward a construction of groups associated to Lie algebras of order three. We will show that it is possible to define an universal enveloping algebra associated to a Lie algebra of order three, and then to endow it with a structure of Hopf algebra.

The content of the paper is the following. In section two the definition of a Lie algebra of order $F$, together with some explicit examples are given. It is shown in section three that one can define a universal enveloping algebra associated to a Lie algebra of order three, and a Poincaré-Birkhoff-Witt theorem is established. It turns out that the PBW basis is strongly related to the three-exterior algebra [26]. In section four the universal enveloping algebra is endowed with a Hopf algebra structure. In section five the study of the Hopf dual enables us to define the parameters of the transformation which turn out to be related to the three-exterior algebra. A conclusion is given in Section VI. Some of the results of this paper was partially given in [27].

It has to be noticed that some attempt to consider a group associated to a Lie algebra of order three $\mathfrak{g}$ has been undertaken in [28], where they have endowed $\mathcal{U}(\mathfrak{g})$ with a Hopf algebra structure and they identified the dual of $\mathcal{U}(\mathfrak{g})$ 
with the group associated with $\mathfrak{g}$. Their construction of Hopf algebra structure on $\mathcal{U}(\mathfrak{g})$ is slightly different from ours since they are considering untwisted Hopf algebra although we are considering twisted Hopf algebras (see Section 4.). They also obtained a result similar to Proposition 2 which enable them to define a group associated to a Lie algebra of order three considering specific polynomials. However, even if their construction is similar to our approach, there is no description of a basis of $\mathcal{U}(\mathfrak{g})$ and the construction of the dual is incomplete.

\section{LIE ALGEBRAS OF ORDER $F$}

In this section we recall the definition and some basic properties of Lie algebras of order $F$ introduced in [14, 15]. Some examples useful for the sequel are also given.

\section{A. Definition and examples of elementary Lie algebras of order three}

We consider $\mathfrak{g}$ a complex vector space and $\varepsilon$ an automorphism of $\mathfrak{g}$ satisfying $\varepsilon^{F}=1$. The vector space $\mathfrak{g}$ is decomposed in $\mathfrak{g}=\mathfrak{g}_{0} \oplus \mathfrak{g}_{1} \cdots \oplus \mathfrak{g}_{F-1}$ where $\mathfrak{g}_{i}$ is the eigenspace corresponding to the eigenvalue $q^{i}$ of $\varepsilon$ where $q=e^{2 i \pi / F}$.

Definition 1 Let $F \in \mathbb{N}^{*}$. A $\mathbb{Z}_{F}$-graded $\mathbb{C}$-vector space $\mathfrak{g}=\mathfrak{g}_{0} \oplus \mathfrak{g}_{1} \oplus \mathfrak{g}_{2} \cdots \oplus \mathfrak{g}_{F-1}$ is called a complex Lie algebra of order $F$ if

1. $\mathfrak{g}_{0}$ is a complex Lie algebra.

2. For all $i=1, \ldots, F-1, \mathfrak{g}_{i}$ is a representation of $\mathfrak{g}_{0}$. If $X \in \mathfrak{g}_{0}, Y \in \mathfrak{g}_{i}$ then $[X, Y]$ denotes the action of $X$ on $Y$ for any $i=1, \cdots, F-1$.

3. For all $i=1, \ldots, F-1$, there exists an $F$-linear, $\mathfrak{g}_{0}$-equivariant map

$$
\{\cdots\}: \mathcal{S}^{F}\left(\mathfrak{g}_{i}\right) \rightarrow \mathfrak{g}_{0}
$$

where $\mathcal{S}^{F}\left(\mathfrak{g}_{i}\right)$ denotes the $F$-fold symmetric product of $\mathfrak{g}_{i}$, satisfying the following (fundamental) identity

$$
\sum_{j=1}^{F+1}\left[\left\{Y_{1}, \ldots, Y_{j-1}, Y_{j+1}, \ldots, Y_{F+1}\right\}, Y_{i}\right]=0
$$

for all $Y_{j} \in \mathfrak{g}_{i}, j=1, . ., F+1$.

Remark 1 If $F=1$, by definition $\mathfrak{g}=\mathfrak{g}_{0}$ and a Lie algebra of order one is a Lie algebra. If $F=2$, then $\mathfrak{g}$ is a Lie superalgebra. Therefore, Lie algebras of order F appear as some kind of generalisations of Lie algebras and superalgebras.

It is important to notice that the bracket $\{\cdots\}$ is a priori not defined for elements in different gradings. This leads to the following definition. 
Proposition 1 Let $\mathfrak{g}=\mathfrak{g}_{0} \oplus \mathfrak{g}_{1} \oplus \cdots \oplus \mathfrak{g}_{F-1}$ be a Lie algebra of order $F$, with $F>1$. For any $i=1, \ldots, F-1$, the $\mathbb{Z}_{F}$-graded vector spaces $\mathfrak{g}_{0} \oplus \mathfrak{g}_{i}$ is a Lie algebra of order $F$. We call these type of algebras elementary Lie algebras of order $F$.

In [15] an inductive process for the construction of Lie algebras of order $F$ starting from a Lie algebra of order $F_{1}$ with $1 \leq F_{1}<F$ is given. In this paper, we restrict ourselves to elementary Lie algebras of order three, $\mathfrak{g}=\mathfrak{g}_{0} \oplus \mathfrak{g}_{1}$. Non-trivial examples of Lie algebras of order $F$ (finite and infinite-dimensional) are given in [14, 15]. We now give some examples of finite-dimensional Lie algebras of order three, which will be relevant in the sequel.

Example 1 Let $\mathfrak{g}$ be any finite-dimensional Lie algebra provided with a symmetric invariant bilinear form (, ) : $\mathfrak{g} \otimes \mathfrak{g} \rightarrow \mathbb{C}$ and define $\mathfrak{g}_{0}=\mathfrak{g}, \mathfrak{g}_{1}=\mathfrak{g}$ with adjoint representation and $\{,$,$\} by$

$$
\left\{Y_{1}, Y_{2}, Y_{3}\right\}=\left(Y_{1}, Y_{2}\right) Y_{3}+\left(Y_{2}, Y_{3}\right) Y_{1}+\left(Y_{1}, Y_{3}\right) Y_{2}
$$

this is a Lie algebra of order three. In particular, if $\mathfrak{g}_{0}$ is a semi-simple Lie algebra and $\mathfrak{g}_{1}=$ ad $\left(\mathfrak{g}_{0}\right)$ with $\left\{J_{a}, a=\right.$ $1, \cdots$, dim $\left.\mathfrak{g}_{0}\right\}$ a basis of $\mathfrak{g}_{0}$ and $\left\{A_{a}, a=1, \cdots\right.$, dim $\left.\mathfrak{g}_{0}\right\}$ the corresponding basis of $\mathfrak{g}_{1}$ we have

$$
\left[J_{a}, J_{b}\right]=f_{a b}^{c} J_{c}, \quad\left[J_{a}, A_{b}\right]=f_{a b}^{c} A_{c}, \quad\left\{A_{a}, A_{b}, A_{c}\right\}=g_{a b} J_{c}+g_{a c} J_{b}+g_{b c} J_{a}
$$

where $g_{a b}=\operatorname{Tr}\left(A_{a} A_{b}\right)$ is the Killing form and $f_{a b}{ }^{c}$ the structure constants of $\mathfrak{g}_{0}$.

Example 2 Let $\mathfrak{g}_{0}=\left\langle L_{\mu \nu}=-L_{\nu \mu}, P_{\mu}, \mu, \nu=0, \cdots, D-1\right\rangle$ be the Poincaré algebra in D-dimensions and $\mathfrak{g}_{1}=$ $\left\langle V_{\mu}, \mu=0, \cdots, D-1\right\rangle$ be the $D$-dimensional vector representation of $\mathfrak{g}_{0}$. The brackets

$$
\begin{aligned}
{\left[L_{\mu \nu}, L_{\rho \sigma}\right] } & =\eta_{\nu \sigma} L_{\rho \mu}-\eta_{\mu \sigma} L_{\rho \nu}+\eta_{\nu \rho} L_{\mu \sigma}-\eta_{\mu \rho} L_{\nu \sigma} \\
{\left[L_{\mu \nu}, P_{\rho}\right] } & =\eta_{\nu \rho} P_{\mu}-\eta_{\mu \rho} P_{\nu},\left[L_{\mu \nu}, V_{\rho}\right]=\eta_{\nu \rho} V_{\mu}-\eta_{\mu \rho} V_{\nu},\left[P_{\mu}, V_{\nu}\right]=0 \\
\left\{V_{\mu}, V_{\nu}, V_{\rho}\right\} & =\eta_{\mu \nu} P_{\rho}+\eta_{\mu \rho} P_{\nu}+\eta_{\rho \nu} P_{\mu}
\end{aligned}
$$

with the metric $\eta_{\mu \nu}=\operatorname{diag}(1,-1, \cdots,-1)$ endow $\mathfrak{g}=\mathfrak{g}_{0} \oplus \mathfrak{g}_{1}$ with an elementary Lie algebra of order three structure which is denoted $\mathfrak{i s o}_{3}(1, D-1)$.

It has been shown that Example 1 (with $\mathfrak{g}_{0}=\mathfrak{s o}(2,3)$ ) and Example 2 (when $D=4$ ) are related through an Inönü-Wigner contraction [15] and a deformation in a Gerstenhaber sense [16].

Example 3 Let $A$ be an associative algebra. We denote by $L(A)$ the Lie algebra defined from $A$ by the bracket $\left[a_{1}, a_{2}\right]=a_{1} a_{2}-a_{2} a_{1}$. We consider the graded vector space $L_{3}(A)=L(A) \oplus A$. We define the following products

$$
\left\{\begin{array}{l}
{\left[a_{1}, a_{2}\right]=a_{1} a_{2}-a_{2} a_{1} \in L(A), \quad \forall a_{1}, a_{2} \in L(A)} \\
{[a, b]=a b-b a \in A, \quad \forall a \in L(A), \forall b \in A} \\
\left\{b_{1}, b_{2}, b_{3}\right\}=\Sigma_{\sigma \in S_{3}} b_{\sigma(1)} b_{\sigma(2)} b_{\sigma(3)} \in L(A), \quad \forall b_{1}, b_{2}, b_{3} \in A .
\end{array}\right.
$$


It is easy to prove that these products provide $L_{3}(A)$ with a structure of elementary Lie algebra of order three. Indeed, one can check that the fundamental identity (1) is just a consequence of the associativity of the product in A. We call this algebra the elementary Lie algebra of order three associated to the associative algebra A.

Given $A$ and $B$ to associative algebras, notice that the application $A \rightarrow L_{3}(A)$ is functorial. Indeed, a morphism of associative algebras $A \rightarrow B$ induces a morphism from $L_{3}(A) \rightarrow L_{3}(B)$. Then, given a Lie algebra of order $3 \mathfrak{g}$ and an associative algebra $A$, there exists a morphism of Lie algebras of order three $\mathfrak{g} \rightarrow L_{3}(A)$ if and only if there exists a linear map $\mathfrak{g} \rightarrow A$ satisfying the conditions in Definition 2. This remark justifies the definition of representation below.

\section{B. Morphisms and representations of an elementary Lie algebras of order $F$}

Let $\mathfrak{g}$ and $\mathfrak{h}$ be two Lie algebras of order $F$. A linear map $f: \mathfrak{g} \longrightarrow \mathfrak{h}$ is a morphism of algebras of order $F$ if $f$ is graded, that is $f=f_{0}+f_{1}+\cdots+f_{F-1}$ with $f_{a}\left(\mathfrak{g}_{a}\right) \subset \mathfrak{h}_{a}$ for all $a \in\{0,1, \cdots, F-1\}$ and

$$
\left\{\begin{array}{l}
f_{a}\left[X, X_{a}\right]=\left[f_{0}(X), f_{a}\left(X_{a}\right)\right], \quad \forall X \in \mathfrak{g}_{0}, X_{a} \in \mathfrak{g}_{a} \\
f_{0}\left\{Y_{1}, \cdots, Y_{F}\right\}=\left\{f_{i}\left(Y_{1}\right), \cdots, f_{i}\left(Y_{F}\right)\right\}, \quad \forall Y_{1}, \cdots, Y_{F} \in \mathfrak{g}_{i}, i=1, \cdots, F-1 .
\end{array}\right.
$$

Let $V$ be a $\mathbb{Z}_{F}-$ graded vector space.

Definition 2 A representation of an elementary Lie algebra of order $F$ is a linear map $\rho: \mathfrak{g}=\mathfrak{g}_{0} \oplus \mathfrak{g}_{1} \rightarrow \operatorname{End}(V)$, such that (for all $X_{i} \in \mathfrak{g}_{0}, Y_{j} \in \mathfrak{g}_{1}$ )

$$
\begin{aligned}
& \rho\left(\left[X_{1}, X_{2}\right]\right)=\rho\left(X_{1}\right) \rho\left(X_{2}\right)-\rho\left(X_{2}\right) \rho\left(X_{1}\right) \\
& \rho\left(\left[X_{1}, Y_{2}\right]\right)=\rho\left(X_{1}\right) \rho\left(Y_{2}\right)-\rho\left(Y_{2}\right) \rho\left(X_{1}\right) \\
& \rho\left(\left\{Y_{1}, \cdots, Y_{F}\right\}\right)=\sum_{\sigma \in S_{F}} \rho\left(Y_{\sigma(1)}\right) \cdots \rho\left(Y_{\sigma(F)}\right)
\end{aligned}
$$

( $S_{F}$ being the group of permutations of $F$ elements).

If $V=V_{0} \oplus \cdots \oplus V_{F-1}$ then for all $a \in\{0, \cdots, F-1\}, V_{a}$ is a $\mathfrak{g}_{0}$-module and we have $\rho\left(\mathfrak{g}_{1}\right)\left(V_{a}\right) \subseteq V_{a+1}$.

Example 4 Let $\mathfrak{g}\left(m_{1}, m_{2}, m_{3}\right)$ and $\mathfrak{g}_{\text {el }}\left(m_{1}, m_{2}, m_{3}\right)$ be the set of $\left(m_{1}+m_{2}+m_{3}\right) \times\left(m_{1}+m_{2}+m_{3}\right)$ matrices of the form

$$
\mathfrak{g}_{\mathrm{el}}\left(m_{1}, m_{2}, m_{3}\right)=\left\{\left(\begin{array}{ccc}
a_{0} & b_{1} & 0 \\
0 & a_{1} & b_{2} \\
b_{0} & 0 & a_{2}
\end{array}\right)\right\}, \mathfrak{g}\left(m_{1}, m_{2}, m_{3}\right)=\left\{\left(\begin{array}{ccc}
a_{0} & b_{1} & c_{2} \\
c_{0} & a_{1} & b_{2} \\
b_{0} & c_{1} & a_{2}
\end{array}\right)\right\}
$$

with $a_{0} \in \mathfrak{g l}\left(m_{1}\right), a_{1} \in \mathfrak{g l}\left(m_{2}\right), a_{3} \in \mathfrak{g l}\left(m_{3}\right), b_{1} \in \mathcal{M}_{m_{1}, m_{2}}(\mathbb{C}), b_{2} \in \mathcal{M}_{m_{2}, m_{3}}(\mathbb{C}), b_{0} \in \mathcal{M}_{m_{3}, m_{1}}(\mathbb{C})$, and $c_{0} \in$ $\mathcal{M}_{m_{2}, m_{1}}(\mathbb{C}), c_{1} \in \mathcal{M}_{m_{3}, m_{2}}(\mathbb{C}), c_{2} \in \mathcal{M}_{m_{1}, m_{3}}(\mathbb{C}) . \quad$ Define $\mathfrak{g}_{0}=\mathfrak{g l}\left(m_{1}\right) \oplus \mathfrak{g l}\left(m_{2}\right) \oplus \mathfrak{g l}\left(m_{3}\right), \mathfrak{g}_{1}=\mathcal{M}_{m_{1}, m_{2}}(\mathbb{C}) \oplus$ 
$\mathcal{M}_{m_{2}, m_{3}}(\mathbb{C}) \oplus \mathcal{M}_{m_{3}, m_{1}}(\mathbb{C})$ and $\mathfrak{g}_{2}=\mathcal{M}_{m_{1}, m_{3}}(\mathbb{C}) \oplus \mathcal{M}_{m_{3}, m_{2}}(\mathbb{C}) \oplus \mathcal{M}_{m_{2}, m_{1}}(\mathbb{C})$. It is obvious that $\mathfrak{g}_{1}$ and $\mathfrak{g}_{2}$ are representations of $\mathfrak{g}_{0}$. Furthermore since $\mathfrak{g}_{i} \mathfrak{g}_{j} \subseteq \mathfrak{g}_{i+j}$ we have $\mathfrak{g}_{1} \mathfrak{g}_{1} \mathfrak{g}_{1} \subseteq \mathfrak{g}_{0}$ and $\mathfrak{g}_{2} \mathfrak{g}_{2} \mathfrak{g}_{2} \subseteq \mathfrak{g}_{0}$ and consequently $\mathfrak{g}\left(m_{1}, m_{2}, m_{3}\right)$ (resp. $\mathfrak{g}_{\text {el }}\left(m_{1}, m_{2}, m_{3}\right)$ ) defines a Lie algebra of order three (resp. an elementary Lie algebra of order three).

\section{UNIVERSAL ENVELOPING ALGEBRA OF LIE ALGEBRAS OF ORDER THREE}

In this section we construct the universal enveloping algebra associated to an elementary Lie algebra of order three. We also show that some Poincaré-Birkhoff-Witt theorem holds true in this context.

\section{A. Enveloping algebra associated to an elementary Lie algebra of order three}

We consider $\mathfrak{g}=\mathfrak{g}_{0} \oplus \mathfrak{g}_{1}$ an elementary Lie algebra of order three and denote generically by $X$ (resp. $Y$ ) the elements of $\mathfrak{g}_{0}$ (resp. $\left.\mathfrak{g}_{1}\right)$.

Definition 3 The universal enveloping algebra $\mathcal{U}(\mathfrak{g})$ is the quotient of the tensor algebra $T(\mathfrak{g})$ by the two sided-ideal generated by

$$
\begin{aligned}
& X_{1} \otimes X_{2}-X_{2} \otimes X_{1}-\left[X_{1}, X_{2}\right], \\
& X_{1} \otimes Y_{2}-Y_{2} \otimes X_{1}-\left[X_{1}, Y_{2}\right] \\
& Y_{1} \otimes Y_{2} \otimes Y_{3}+Y_{3} \otimes Y_{1} \otimes Y_{2}+Y_{2} \otimes Y_{3} \otimes Y_{1}+Y_{1} \otimes Y_{3} \otimes Y_{2}+Y_{3} \otimes Y_{2} \otimes Y_{1}+Y_{2} \otimes Y_{1} \otimes Y_{3}-\left\{Y_{1}, Y_{2}, Y_{3}\right\} .
\end{aligned}
$$

As before, the fundamental identity (1) is a direct consequence of the associativity of the tensorial product in $T(\mathfrak{g})$. We now state the universal property of $\mathcal{U}(\mathfrak{g})$.

Theorem 1 Let $\mathfrak{g}$ be an elementary Lie algebra of order three. Given any associative algebra $A$ and any morphism $f$ of Lie algebras of order three from $\mathfrak{g}$ to $L_{3}(A)$, there exists an unique morphism of algebras $\varphi: \mathcal{U}(\mathfrak{g}) \longrightarrow A$ such that $\varphi \circ i_{\mathfrak{g}}=f$ where $i_{\mathfrak{g}}$ is the composition of the canonical injection of $\mathfrak{g}$ into $T(\mathfrak{g})$ and the canonical surjection of $T(\mathfrak{g})$ into $\mathcal{U}(\mathfrak{g})$.

Proof By definition of the tensor algebra, $f$ extends to a morphism of algebras $\tilde{f}$ from $T(\mathfrak{g})$ to $A$ defined by $\tilde{f}\left(X_{1} \otimes X_{2}\right)=f_{0}\left(X_{1}\right) f_{0}\left(X_{2}\right), \tilde{f}\left(X_{1} \otimes Y\right)=f_{0}\left(X_{1}\right) f_{1}(Y)$, etc., for $X_{1}, X_{2} \in \mathfrak{g}_{0}$ and $Y \in \mathfrak{g}_{1}$. This map satisfies

$$
\tilde{f}\left(X \otimes X^{\prime}-X^{\prime} \otimes X-\left[X, X^{\prime}\right]\right)=\left[f_{0}(X), f_{0}\left(X^{\prime}\right)\right]-f_{0}\left[X, X^{\prime}\right]=0, \forall X, X^{\prime} \in \mathfrak{g}_{0}
$$

because $f_{0}$ is morphism of Lie algebras. Likewise we have

$$
\tilde{f}(X \otimes Y-Y \otimes X-[X, Y])=\left[f_{0}(X), f_{1}(Y)\right]-f_{1}[X, Y]=0, \forall X \in \mathfrak{g}_{0}, Y \in \mathfrak{g}_{1}
$$


and

$$
\tilde{f}\left(\Sigma Y_{1} \otimes Y_{2} \otimes Y_{3}-\left\{Y_{1}, Y_{2}, Y_{3}\right\}\right)=\left\{f_{1}\left(Y_{1}\right), f_{1}\left(Y_{2}\right), f_{1}\left(Y_{3}\right)\right\}-f_{0}\left\{Y_{1}, Y_{2}, Y_{3}\right\}=0
$$

for any $Y_{1}, Y_{2}, Y_{3} \in \mathfrak{g}_{1}$ where $\Sigma Y_{1} \otimes Y_{2} \otimes Y_{3}$ is the symmetric product. Then $\tilde{f}$ is trivial on the ideal generated by the relation defining $\mathcal{U}(\mathfrak{g})$. This proves the existence of $\varphi$. The uniqueness is due to the fact that $\mathfrak{g}$ generates the algebra $T(\mathfrak{g})$. Q.E.D.

\section{B. The Poincaré-Birkhoff-Witt theorem}

Having defined the universal enveloping algebra, we now establish the Poincaré-Birkhoff-Witt theorem. However, it is necessary to recall firstly some results concerning the $n$-exterior algebra, that becomes central in this context.

\section{The n-exterior Roby algebra}

Let $n \geq 2$ be an integer. The $n$-exterior algebra has been introduced by Roby [26] (see also [8, 29]). In this section we recall some of the main results of [26] useful for this paper. Let $V$ be a $d$-dimensional vector space over the field $\mathbb{C}$ (or $\mathbb{R}$ ) and consider $T(V)=\mathbb{C} \oplus V \oplus(V \otimes V) \oplus V^{\otimes^{3}} \oplus \cdots$ the tensor algebra over $V$. Consider $v_{1}, v_{2}, \cdots, v_{p}, p$ different independent elements of $V$ and $n_{1}, \cdots, n_{p} \in \mathbb{N}^{*}$ such that $n_{1}+\cdots n_{p}=n$. Define the tensor $\sigma=\mathcal{S}\left(v_{1}^{\otimes^{n_{1}}} \otimes \cdots \otimes v_{p}^{\otimes^{n_{p}}}\right)$, with $\mathcal{S}$ being the symmetrised tensorial product of the tensor $v_{1}^{\otimes^{n_{1}}} \otimes \cdots \otimes v_{p}^{\otimes^{n_{p}}}$. This symmetrised tensor contains $\frac{n !}{n_{1} ! \cdots n_{p} !}$ monomials. For instance $\mathcal{S}\left(v_{1}^{\otimes^{2}} \otimes v_{2}\right)=v_{1}^{\otimes^{2}} \otimes v_{2}+v_{1} \otimes v_{2} \otimes v_{1}+v_{2} \otimes v_{1}^{\otimes^{2}}$. We consider $\mathcal{I}(V, n)$ the two-sided ideal generated by elements of the form $\sigma$.

Definition 4 Let $V$ be a d-dimensional vector space and let $\mathcal{I}(V, n)$ be defined as above. The $n$-exterior algebra is the $\mathbb{Z}_{n}$-graded vector space $\Lambda(V, n)=T(V) / \mathcal{I}(V, n)$.

Remark 2 The composition of the natural map $V \rightarrow T(V)$ with the canonical projection $T(V) \rightarrow \Lambda(V, n)$ gives $V \subset \Lambda(V, n)$ and we identify $V$ with its image under this map. Thus, if we denote $\left\{e_{i}, 1 \leq i \leq d\right\}$ a basis of $V$ the algebra $\Lambda(V, n)$ can be equivalently defined by generators and relations:

$$
\left\{e_{i_{1}}, \cdots, e_{i_{n}}\right\}=\sum_{\tau \in S_{n}} e_{i_{\tau(1)}} \cdots e_{i_{\tau(n)}}=0,
$$

with $S_{n}$ the group of permutations with $n$ elements.

If we define $\Lambda(V, n)_{i}$ the set of elements of degree $i(\bmod . n)$ we have $\Lambda(V, n)_{i} \Lambda(V, n)_{j} \subseteq \Lambda(V, n)_{i+j}(\bmod . n)$ and

$$
\Lambda(V, n)=\Lambda(V, n)_{0} \oplus \cdots \oplus \Lambda(V, n)_{n-1}
$$


thus $\Lambda(V, n)$ is a $\mathbb{Z}_{n}$-graded vector space. When $n=2, \Lambda(V, 2)$ coincides with the usual exterior algebra. But when $n>2, \Lambda(V, n)$ is very different from the exterior algebra. Indeed, $\Lambda(V, n)$ is defined through $n$-th order relations, and consequently the number of independent monomials increases with polynomial's degree (for instance, $\left(e_{1} e_{2}\right)^{k}, k \geq 0$ are all independent). This means that we do not have enough constraints among the generators to order them in some fixed way and, as a consequence, $\Lambda(V, n)$ turns out to be an infinite-dimensional algebra. In particular if we define $\Lambda_{k}(V, n)=V^{\otimes^{k}} / \mathcal{I}(V, n)$, we have $\Lambda_{0}(V, n)=\mathbb{C}, \Lambda_{1}(V, n)=V, \cdots, \Lambda_{n-1}(V, n)=V^{\otimes^{n-1}}$, but the spaces $\Lambda_{k}(V, n), k \geq n$ are not empty and more difficult to characterise. In order to construct a basis of $\Lambda(V, n)$, i.e. to specify $\Lambda_{k}(V, n), k \geq n$ we need one more definition.

Definition 5 We say that the sequence $\left(i_{1}, i_{2}, \cdots i_{k}\right), k \geq n$ with $i_{1}, \cdots, i_{k} \in\{1, \cdots, d\}$ has a rise of length $n$ if it exists $0 \leq \ell \leq k-n$ such that $i_{\ell+1} \leq i_{\ell+2} \leq \cdots \leq i_{\ell+n}$. We denote by $I_{k, n} \subseteq\{1, \cdots, d\}^{k}$ the set of $k$ indices which has a rise of length $n$.

Theorem 2 (N. Roby [26]) A basis of $\Lambda(V, n)$ is given by the words $e_{i_{1}} \cdots e_{i_{k}}, k \in \mathbb{N}$ such that the sequence $\left(i_{1}, \cdots, i_{k}\right)$ has not a rise of length $n$ i.e. $\left(i_{1}, \cdots, i_{k}\right) \in\{1, \cdots, d\}^{k} \backslash I_{k, n}$. The sequence $I=\left(i_{1}, \cdots, i_{n}\right)$ will be called a Roby sequence, and the element $e_{I}=e_{i_{1}} \cdots e_{i_{n}}$ a Roby element or a Roby word.

Example 5 1. If $\operatorname{dim} V=2$ a basis of $\Lambda(V, 3)$ is given by the elements $t\left(e_{2} e_{1}\right)^{k} t^{\prime}, k \in \mathbb{N}, t, t^{\prime}=1, e_{1}, e_{2}$ [26]. This means that

$$
\begin{aligned}
\Lambda_{0}(V, 3) & =\mathbb{C} \\
\Lambda_{1}(V, 3) & =V \\
\Lambda_{2}(V, 3) & =V \otimes V \\
\Lambda_{2 n+1}(V, 3) & =\left\langle e_{i}\left(e_{2} e_{1}\right)^{n},\left(e_{2} e_{1}\right)^{n} e_{i}, i=1,2,\right\rangle, n \geq 1 \\
\Lambda_{2 n}(V, 3) & =\left\langle e_{i}\left(e_{2} e_{1}\right)^{n-1} e_{j}, i, j=1,2,\left(e_{2} e_{1}\right)^{n}\right\rangle, n \geq 2 .
\end{aligned}
$$

2. If $\operatorname{dim} V=4$ then $\operatorname{dim} \Lambda_{3}(V, 3)=44$, the basis is given by

$$
\begin{array}{lc}
e_{i} e_{j} e_{i}, e_{j} e_{i} e_{i}, & i<j, \\
e_{i} e_{i} e_{j}, e_{i} e_{j} e_{i}, & i>j, \\
e_{j} e_{k} e_{i}, e_{k} e_{i} e_{j}, e_{i} e_{k} e_{j}, e_{j} e_{i} e_{k}, e_{k} e_{j} e_{i}, & i<j<k .
\end{array}
$$

Similarly $\operatorname{dim} \Lambda_{4}(V, 3)=256$. 
2. A Poincaré-Birkhoff-Witt basis of $\mathcal{U}(\mathfrak{g})$

Let $\mathfrak{g}=\mathfrak{g}_{0} \oplus \mathfrak{g}_{1}$ be an elementary Lie algebra of order three. We denote by the letters $X$ (resp. $Y$ ) the elements of $\mathfrak{g}_{0}$ (resp. $\mathfrak{g}_{1}$ ). Then an element of $\mathcal{U}(\mathfrak{g})$ is a word written using letters as $X_{\alpha}$ and $Y_{\beta}$ belonging to the free algebra $\mathcal{L}(X, Y)$ generated by $\left\{X_{1}, \ldots, X_{p}, Y_{1}, \ldots, Y_{q}\right\}$, where $p=\operatorname{dim} \mathfrak{g}_{0}$ and $q=\operatorname{dim} \mathfrak{g}_{1}$. We have the following three rules of reduction of a word:

- $Y X=X Y-[X, Y]$. As $[X, Y] \in \mathfrak{g}_{1}$, this rule permits to reduce a word as $m(X) m(Y)$ where $m(X)($ resp. $m(Y))$ contains only $X$ (resp. $Y$ ).

- $X_{2} X_{1}=X_{1} X_{2}-\left[X_{1}, X_{2}\right]$. As $\left[X_{1}, X_{2}\right] \in \mathfrak{g}_{0}$, this rule permits to write a word $m(X)$ on the classical PoincaréBirkhoff-Witt words of $\mathcal{U}\left(\mathfrak{g}_{0}\right)$.

- $Y_{a} Y_{b} Y_{c}=-Y_{b} Y_{a} Y_{c}-Y_{c} Y_{b} Y_{a}-Y_{a} Y_{c} Y_{b}-Y_{b} Y_{c} Y_{a}-Y_{c} Y_{a} Y_{b}-\left\{Y_{a}, Y_{b}, Y_{c}\right\}$. If $\left\{Y_{a}, Y_{b}, Y_{c}\right\}=0$ for all $a, b, c$, this rule permits to write a word $m(Y)$ as a Roby word of the three-exterior algebra $\Lambda\left(\mathfrak{g}_{1}, 3\right)$.

The first two rules permits to write a word $m(X, Y)$ of the free algebra $\mathcal{L}(X, Y)$ on the form $m_{1}(X) m_{2}(Y)$ with $m_{1}(X) \in \mathcal{U}\left(\mathfrak{g}_{0}\right)$ and $m_{2}(Y) \in \mathcal{L}(Y)$. Then we have to reduce $m_{2}(Y)$. Suppose that $m(Y)$ is a word of length $n$. We will denote it by $m^{n}(Y)$. If this word is a Roby word, the reduction is finished. If not, there exist in $m^{n}(Y)=Y_{a_{1}} Y_{a_{2}} \ldots Y_{a_{n}}$ a triple $\left(a_{i} \leq a_{i+1} \leq a_{i+2}\right)$ such that $\left(a_{1}, \cdots, a_{n}\right)$ is not a Roby sequence. In this case, the rule 3 permits to write

$$
m^{n}(Y)=\sum m_{i}^{n}(Y)+m^{n-2}(X, Y)
$$

where $m^{n-2}(X, Y) \in \mathcal{L}(X, Y)$ but it is of length $n-2$. Let $\mathcal{L}_{n-2}(X, Y)$ be the linear subspace of $\mathcal{L}(X, Y)$ whose element are of length smaller or equal than $n-2$. Then $m^{n}(Y)=\sum m_{i}^{n}(Y)$ modulo $\mathcal{L}_{n-2}(X, Y)$. We can apply the Roby rule 3 to obtain $m^{n}(Y)=\sum m_{\alpha}^{n}(Y)$ modulo $\mathcal{L}_{n-2}(X, Y)$ where $m_{\alpha}^{n}(Y)$ are Roby words. Now we have to consider words of type $m^{n-2}(X, Y)$ of length $n-2$. The first two rules reduces such a word to $m_{1}(X) m_{2}(Y)$ with $m_{1}(X) \in \mathcal{U}\left(\mathfrak{g}_{0}\right)$ and $m_{2}(Y) \in \mathcal{L}(Y)$ but $m_{2}(Y)$ is a word of length smaller than $n-2$. An induction process permits to conclude.

Theorem 3 The universal algebra is generated by a basis of the universal enveloping algebra $\mathcal{U}\left(\mathfrak{g}_{0}\right)$ and by a Roby basis of the three-exterior algebra $\Lambda\left(\mathfrak{g}_{1}, 3\right)$. Then, as vector space, we have

$$
\mathcal{U}(\mathfrak{g})=\mathcal{U}\left(\mathfrak{g}_{0}\right) \otimes \Lambda\left(\mathfrak{g}_{1}, 3\right)
$$

This result has been conjectured in [15].

In the usual way, the representations of $\mathfrak{g}$ are in bijective correspondence with the representations of the associative algebra $\mathcal{U}(\mathfrak{g})$. Consequently, if $I \subset \mathcal{U}(\mathfrak{g})$ is a two-sided ideal, then the quotient $\mathcal{U}(\mathfrak{g}) / I$ gives a 
representation of $\mathfrak{g}$. If $\mathfrak{g}=\mathfrak{g}_{0} \oplus \mathfrak{g}_{1}$, with $\mathfrak{g}_{0}$ a semi-simple Lie algebra, we decompose $\mathfrak{g}$ in its Borel form $\mathfrak{g}=\mathfrak{n}_{+} \oplus \mathfrak{h} \oplus \mathfrak{n}_{-}=\left(\mathfrak{n}_{0+} \oplus \mathfrak{h}_{0+} \oplus \mathfrak{n}_{0-}\right) \oplus\left(\mathfrak{n}_{1+} \oplus \mathfrak{h}_{1+} \oplus \mathfrak{n}_{1-}\right)$. If we assume that some highest weight representation can be obtained since the algebra $\mathfrak{g}$ is defined by cubic relations, the representation so obtained will be infinitedimensional. This can be compare with the Roby Theorem 2 for the three-exterior algebra. This means that in finite-dimensional representations of $\mathfrak{g}$, as in the Example 4 the elements of $\mathfrak{n}_{-}$are not only nilpotent but also satisfy additional relations and the representation is non-faithful. See [15] for more details.

\section{Example : $\mathcal{U}\left(\mathfrak{i s o}_{3}(1,3)\right)$.}

We consider the Lie algebra of order three given in Example 2. Introduce $X_{a}=\left(L_{01}, L_{02}, L_{03}, L_{12}, L_{13}, L_{23}\right.$, $\left.P_{0}, P_{1}, P_{2}, P_{3}\right)$ the generators of $\mathfrak{i s o}(1,3)$ (we restrict ourselves to $D=4$ since the generic case is analogous), and for any $\vec{a}=\left(a_{1}, \cdots, a_{10}\right) \in \mathbb{N}^{10}$ set

$$
X_{\vec{a}}=\frac{X_{1}^{a_{1}}}{a_{1} !} \cdots \frac{X_{10}^{a_{1}}}{a_{10} !} .
$$

Consider now $I_{\ell}=\left(i_{\mu_{1}}, \cdots, i_{\mu_{\ell}}\right) \in\{0,1,2,3\}^{\ell} \backslash I_{\ell, 3}$ a Roby sequence (see Definition 5) and define

$$
V_{\left(\mu_{1}, \cdots, \mu_{\ell}\right)}=V_{\mu_{1}} \cdots V_{\mu_{\ell}}
$$

From Theorem 3, the Poincaré-Birkhoff-Witt basis is given by

$$
\left\{g_{\vec{b},\left(\mu_{1}, \cdots, \mu_{\ell}\right)}=X_{\vec{b}} V_{\left(\mu_{1}, \cdots, \mu_{\ell}\right)}, \vec{b} \in \mathbb{N}^{10}, \ell \in \mathbb{N},\left(\mu_{1}, \cdots, \mu_{\ell}\right) \in\{0,1,2,3\}^{\ell} \backslash I_{\ell, 3}\right\} .
$$

Here we denote by 1 the neutral element.

The algebra structure constructed on $\mathcal{U}\left(\mathfrak{i s o}_{3}(1,3)\right)$ is immediate, and for instance we have: $X_{\vec{b}} V_{\left(\mu_{1}, \cdots, \mu_{\ell}\right)}=$ $g_{\vec{b},\left(\mu_{1}, \cdots, \mu_{\ell}\right)}$. The multiplication law for $X_{\vec{a}}$ and $X_{\vec{b}}$ uses explicitly the commutation relation of the algebra iso $(1,3)$ and for instance $X_{a} X_{b}=X_{b} X_{a}+\left[X_{a}, X_{b}\right]$ if $a>b$. But the multiplication rule for $V_{\left(\mu_{1}, \cdots, \mu_{\ell}\right)}$ and $V_{\left(\nu_{1}, \cdots, \nu_{\ell^{\prime}}\right)}$ is more involved. Indeed, if $\left(\mu_{1}, \cdots, \mu_{\ell}\right) \in\{0,1,2,3\}^{\ell} \backslash I_{\ell, 3}$ and $\left(i_{\nu_{1}}, \cdots, \nu_{\ell^{\prime}}\right) \in\{0,1,2,3\}^{\ell^{\prime}} \backslash I_{\ell^{\prime}, 3}$ this does not mean that $\left(i_{\mu_{1}}, \cdots, i_{\mu_{\ell}}, i_{\nu_{1}}, \cdots, i_{\nu_{\ell^{\prime}}}\right) \in\{0,1,2,3\}^{\ell+\ell^{\prime}} \backslash I_{\ell+\ell^{\prime}, 3}$. For instance, since (112) is not a Roby sequence, we have $V_{1} V_{(12)}=-\frac{1}{2} P_{2}-V_{(121)}-V_{(211)}$, for the algebra of Example 2 


\section{HOPF STRUCTURE ON $\mathcal{U}(\mathfrak{g})$}

Let $\mathcal{U}(\mathfrak{g})$ be the universal enveloping algebra associated to $\mathfrak{g}$ a Lie algebra of order three. We consider on $\mathfrak{g}$ a $\mathbb{Z}_{3}$ - grading coming from the decomposition of the structure of Lie algebra of order three. As $\mathfrak{g}$ is an elementary Lie algebra of order three, we can consider $\mathfrak{g}_{0}$ with grading 0 and $\mathfrak{g}_{1}$ with grading 1 . Let $q$ be a cubic primitive root of unity. We consider on $\mathcal{U}(\mathfrak{g}) \otimes \mathcal{U}(\mathfrak{g})$ a structure of algebra given by

$$
(a \otimes c) \bullet(b \otimes d)=q^{|b||c|} a b \otimes c d
$$

where $|x|$ denotes the grading of an homogeneous element $x$. This associative structure on $\mathcal{U}(\mathfrak{g}) \otimes \mathcal{U}(\mathfrak{g})$ will be denoted by $\mathcal{U}(\mathfrak{g}) \underline{\otimes \mathcal{U}}(\mathfrak{g})$. We consider

$$
\bar{\Delta}: T(\mathfrak{g}) \rightarrow \mathcal{U}(\mathfrak{g}) \underline{\otimes} \mathcal{U}(\mathfrak{g})
$$

defined by

- $\bar{\Delta}(1)=1 \otimes 1$,

- $\bar{\Delta}(X)=1 \otimes X+X \otimes 1$,

- $\bar{\Delta}(Y)=1 \otimes Y+Y \otimes 1$,

and

- $\bar{\Delta}(a \otimes b)=\sum q^{\left|a_{(2)}\right|\left|b_{(1)}\right|} a_{(1)} b_{(1)} \otimes a_{(2)} b_{(2)}=\bar{\Delta}(a) \bullet \bar{\Delta}(b)$,

for all $X \in \mathfrak{g}_{0}, Y \in \mathfrak{g}_{1}$ and $a, b \in T(\mathfrak{g})$.

Lemma 1 If we consider $L_{3}(\mathcal{U}(\mathfrak{g}) \underline{\otimes} \mathcal{U}(\mathfrak{g}))$ the Lie algebra of order 3 associated to the associative algebra $\mathcal{U}(\mathfrak{g}) \underline{\otimes U}(\mathfrak{g})$, then

$$
\bar{\Delta}([X, G])=[\bar{\Delta}(X), \bar{\Delta}(G)]_{L_{3}(\mathcal{U}(\mathfrak{g}) \underline{\otimes} \mathcal{U}(\mathfrak{g}))}
$$

and

$$
\bar{\Delta}\left\{Y_{1}, Y_{2}, Y_{3}\right\}=\left\{\bar{\Delta}\left(Y_{1}\right), \bar{\Delta}\left(Y_{2}\right), \bar{\Delta}\left(Y_{3}\right)\right\}_{L_{3}((\mathcal{U}(\mathfrak{g}) \underline{\otimes} \mathcal{U}(\mathfrak{g}))}
$$

with $X \in \mathfrak{g}_{0}, G \in \mathfrak{g}$ and $Y_{i} \in \mathfrak{g}_{1}$ and where $[,]_{L_{3}(\mathcal{U}(\mathfrak{g}) \underline{\otimes} \mathcal{U}(\mathfrak{g}))}$ and $\{,,\}_{L_{3}(\mathcal{U}(\mathfrak{g}) \underline{\otimes} \mathcal{U}(\mathfrak{g}))}$ denotes the bracket and the symmetric product of the Lie algebra of order three, $L_{3}(\mathcal{U}(\mathfrak{g}) \underline{\otimes \mathcal{U}}(\mathfrak{g}))$.

Proof As $\mathcal{U}(\mathfrak{g}) \underline{\otimes U}(\mathfrak{g})$ is a graded associative algebra, we consider the associated structure of Lie algebra of order three. Let us look the second identity. We have $\bar{\Delta}\left(Y_{i}\right)=1 \otimes Y_{i}+Y_{i} \otimes 1$. On $L_{3}(\mathcal{U}(\mathfrak{g}) \otimes \mathcal{U}(\mathfrak{g}))$ we consider the product

$$
\left\{b_{1}, b_{2}, b_{3}\right\}_{L_{3}(\mathcal{U}(\mathfrak{g}) \underline{\otimes} \mathcal{U}(\mathfrak{g}))}=\Sigma_{\sigma \in S_{3}} b_{\sigma(1)} \bullet b_{\sigma(2)} \bullet b_{\sigma(3)}, \quad \forall b_{1}, b_{2}, b_{3} \in \mathcal{U}(\mathfrak{g}) \underline{\otimes \mathcal{U}}(\mathfrak{g}) .
$$


We deduce

$$
\left\{\bar{\Delta}\left(Y_{1}\right), \bar{\Delta}\left(Y_{2}\right), \bar{\Delta}\left(Y_{2}\right)\right\}_{L_{3}(\mathcal{U}(\mathfrak{g}) \underline{\otimes} \mathcal{U}(\mathfrak{g}))}=\left\{1 \otimes Y_{1}+Y_{1} \otimes 1,1 \otimes Y_{2}+Y_{2} \otimes 1,1 \otimes Y_{3}+Y_{3} \otimes 1\right\}_{L_{3}(\mathcal{U}(\mathfrak{g}) \underline{\otimes} \mathcal{U}(\mathfrak{g}))} .
$$

We have to compute the following products $\left\{1 \otimes Y_{1}, 1 \otimes Y_{2}, 1 \otimes Y_{3}\right\}_{L_{3}(\mathcal{U}(\mathfrak{g}) \underline{\otimes}(\mathfrak{G}))},\left\{Y_{1} \otimes 1, Y_{2} \otimes 1, Y_{3} \otimes 1\right\}_{L_{3}(\mathcal{U}(\mathfrak{g}) \underline{\otimes}(\mathfrak{g}))}$, and the crossed products of the form $\left\{1 \otimes Y_{1}, 1 \otimes Y_{2}, Y_{3} \otimes 1\right\}_{L_{3}(\mathcal{U}(\mathfrak{g}) \otimes \mathcal{U}(\mathfrak{g}))},\left\{1 \otimes Y_{1}, Y_{2} \otimes 1, Y_{3} \otimes 1\right\}_{L_{3}(\mathcal{U}(\mathfrak{g}) \otimes \mathcal{U}(\mathfrak{g}))}$ etc. By definition

$\left\{1 \otimes Y_{1}, 1 \otimes Y_{2}, 1 \otimes Y_{3}\right\}_{L_{3}(\mathcal{U}(\mathfrak{g}) \underline{\otimes}(\mathfrak{U}))}=\Sigma_{\sigma \in S_{3}} 1 \otimes Y_{\sigma(1)} \bullet 1 \otimes Y_{\sigma(2)} \bullet 1 \otimes Y_{\sigma(3)}=\Sigma_{\sigma \in S_{3}} 1 \otimes Y_{\sigma(1)} Y_{\sigma(2)} Y_{\sigma(3)}=1 \otimes\left\{Y_{1}, Y_{2}, Y_{3}\right\}$.

Similarly

$\left\{Y_{1} \otimes 1, Y_{2} \otimes 1, Y_{3} \otimes 1\right\}_{L_{3}(\mathcal{U}(\mathfrak{g}) \underline{\otimes}(\mathfrak{g}))}=\Sigma_{\sigma \in S_{3}} Y_{\sigma(1)} \otimes 1 \bullet Y_{\sigma(2)} \otimes 1 \bullet Y_{\sigma(3)} \otimes 1=\Sigma_{\sigma \in S_{3}} Y_{\sigma(1)} Y_{\sigma(2)} Y_{\sigma(3)} \otimes 1=\left\{Y_{1}, Y_{2}, Y_{3}\right\} \otimes 1$.

For the crossed terms, we have

$$
\begin{aligned}
\left\{1 \otimes Y_{1}, 1 \otimes Y_{2}, Y_{3} \otimes 1\right\}_{L_{3}(\mathcal{U}(\mathfrak{g}) \underline{\otimes}(\mathfrak{U}(\mathfrak{g}))} & =1 \otimes Y_{1} \bullet 1 \otimes Y_{2} \bullet Y_{3} \otimes 1+1 \otimes Y_{2} \bullet 1 \otimes Y_{1} \bullet Y_{3} \otimes 1+Y_{3} \otimes 1 \bullet 1 \otimes Y_{2} \bullet 1 \otimes Y_{1} \\
& +1 \otimes Y_{1} \bullet Y_{3} \otimes 1 \bullet 1 \otimes Y_{2}+1 \otimes Y_{2} \bullet Y_{3} \otimes 1 \bullet 1 \otimes Y_{1}+Y_{3} \otimes 1 \bullet 1 \otimes Y_{1} \bullet 1 \otimes Y_{2} \\
& =\left(1+q+q^{2}\right)\left(Y_{3} \otimes Y_{1} Y_{2}+Y_{3} \otimes Y_{2} Y_{1}\right) \\
& =0 .
\end{aligned}
$$

Then

$$
\begin{aligned}
& \left\{\bar{\Delta}\left(Y_{1}\right), \bar{\Delta}\left(Y_{2}\right), \bar{\Delta}\left(Y_{2}\right)\right\}_{L_{3}(\mathcal{U}(\mathfrak{g}) \underline{\otimes}(\mathfrak{U}))}=\left\{1 \otimes Y_{1}, 1 \otimes Y_{2}, 1 \otimes Y_{3}\right\}_{\mathcal{U}(\mathfrak{g}) \underline{\otimes}(\mathfrak{g})}+\left\{Y_{1} \otimes 1, Y_{2} \otimes 1, Y_{3} \otimes 1\right\}_{\mathcal{U}(\mathfrak{g}) \underline{\otimes}(\mathfrak{g})} \\
& =1 \otimes\left\{Y_{1}, Y_{2}, Y_{3}\right\}+\left\{Y_{1}, Y_{2}, Y_{3}\right\} \otimes 1 \\
& =\bar{\Delta}\left\{Y_{1}, Y_{2}, Y_{3}\right\} \text {. }
\end{aligned}
$$

Similarly, we prove that

$$
\left[\bar{\Delta}\left(X_{1}\right), \bar{\Delta}\left(X_{2}\right)\right]_{L_{3}(\mathcal{U}(\mathfrak{g}) \underline{\otimes} \mathcal{U}(\mathfrak{g}))}=\bar{\Delta}\left[X_{1}, X_{2}\right]
$$

Q.E.D.

From this lemma, we deduce that $\operatorname{Ker}(\bar{\Delta})$ contains the ideal defining $\mathcal{U}(\mathfrak{g})$. Then applying Theorem $\mathbb{1}, \bar{\Delta}$ induces a comultiplication

$$
\Delta: \mathcal{U}(\mathfrak{g}) \rightarrow \mathcal{U}(\mathfrak{g}) \underline{\otimes} \mathcal{U}(\mathfrak{g}) .
$$

The elements satisfying $\Delta(u)=1 \otimes u+u \otimes 1$ are called primitive. The comultiplication is coassociative

$$
(\Delta \otimes I d) \circ \Delta=(I d \otimes \Delta) \circ \Delta .
$$


In fact $(\Delta \otimes I d) \circ \Delta(a b)=(\Delta \otimes I d) \circ(\Delta(a) \bullet \Delta(b))=((\Delta \otimes I d) \circ \Delta(a)) \bullet((\Delta \otimes I d) \circ \Delta(b))$. This last relation comes from the fact that the coproduct $\Delta$ is of degree 0 . It satisfies also $\Delta([X, G])=\Delta(X G)-\Delta(G X)=1 \otimes X G+X \otimes$ $G+G \otimes X+X G \otimes 1-1 \otimes G X-G \otimes X-X \otimes G-G X \otimes 1$ that is

$$
\Delta([X, G])=[X, G] \otimes 1+1 \otimes[X, G]
$$

for all $(X, G) \in \mathfrak{g}_{0} \times \mathfrak{g}$ that is $\left[X_{1}, X_{2}\right]$ and $[X, Y]$ are primitive elements. Likewise, if $Y_{1}, Y_{2}, Y_{3} \in \mathfrak{g}_{1}$, then

$$
\Delta\left\{Y_{1}, Y_{2}, Y_{3}\right\}=\sum_{\sigma \in S_{3}} \Delta\left(Y_{\sigma(1)} Y_{\sigma(2)} Y_{\sigma(3)}\right)=\left\{Y_{1}, Y_{2}, Y_{3}\right\} \otimes 1+1 \otimes\left\{Y_{1}, Y_{2}, Y_{3}\right\} .
$$

Thus $\left\{Y_{1}, Y_{2}, Y_{3}\right\}$ is also a primitive element. This means that the elements of $\mathfrak{g}$ are primitive elements.

The counity

$$
\varepsilon: \mathcal{U}(\mathfrak{g}) \rightarrow \mathbb{C}
$$

is the morphism given from $\varepsilon(1)=1, \varepsilon(X)=\varepsilon(Y)=0$ and the antipode is the $\mathbb{Z}_{3}$ anti-homomorphism

$$
S: \mathcal{U}(\mathfrak{g}) \rightarrow \mathcal{U}(\mathfrak{g})
$$

given from

$$
S(1)=1, S(X)=-X, S(Y)=-Y
$$

and satisfying

$$
S(a b)=q^{|a||b|} S(b) S(a)
$$

Definition 6 We call Hopf structure associated to a Lie algebra $\mathfrak{g}$ of order three, the Hopf structure on the enveloping algebra $\mathcal{U}(\mathfrak{g})$ given by $(\Delta, \varepsilon, S)$.

As a final remark for this subsection it should be mentioned that these twisted Hopf algebras (defined by a twisted tensorial product) has been introduced by Majid [25] in order to describe $q$-deformations and braiding structures. He called them anyonic Hopf algebras. It as to be stressed that the structures we are considering have a priori nothing to do with braiding and $q$-deformations, even if they share some similarities. It should also be mentioned that a different Hopf algebra associated to Lie algebras of order three has been defined in [28], where the coproduct was defined by the usual tensorial product and the twist (necessary to ensure (8) ) was generated by an additional element of order three, the grading map $\varepsilon$. In fact these two structures are related by the transmutation theorem that maps an anyonic Hopf algebra to a an (untwisted) Hopf algebra [25].

Example 6 For $\mathcal{U}\left(\mathfrak{i s o}_{3}(1,4)\right)$ the coproduct is given by 


$$
\begin{aligned}
\Delta X_{\vec{a}} & =\sum_{\vec{b}+\vec{c}=\vec{a}} V_{\vec{b}} \otimes V_{\vec{c}} \\
\Delta V_{I_{\ell}} & =\sum_{I_{\ell^{\prime}}+I_{\ell^{\prime \prime}}=I_{\ell}} q^{-N\left(\left(I_{\ell^{\prime}}, I_{\ell^{\prime \prime}}\right), I_{\ell}\right)} V_{I_{\ell^{\prime}}} \otimes V_{I_{\ell^{\prime \prime}}},
\end{aligned}
$$

where $I_{\ell}=\left(\mu_{1}, \cdots, \mu_{\ell}\right) \in\{0,1,2,3\}^{\ell} \backslash I_{\ell, 3}$ and the sum is taken over all complementary subsequences of $I_{\ell} I_{\ell^{\prime}}=$ $\left(\nu_{1}, \cdots, \nu_{\ell^{\prime}}\right)$ and $I_{\ell^{\prime \prime}}=\left(\rho_{1}, \cdots, \rho_{\ell^{\prime \prime}}\right)$ with $\ell=\ell^{\prime}+\ell^{\prime \prime}$. In this sum, $N\left(\left(I_{\ell^{\prime}}, I_{\ell^{\prime \prime}}\right), I_{\ell}\right)$ represents the number of successive transpositions which bring $\left(\nu_{1}, \cdots, \nu_{\ell^{\prime}}, \rho_{1}, \cdots, \rho_{\ell^{\prime \prime}}\right)$ into the ordered sequence $\left(\mu_{1}, \cdots, \mu_{\ell}\right)$ transposing firstly $\rho_{1}$, then $\rho_{2}$ and finally $\rho_{\ell^{\prime \prime}}$. For instance this factor becomes 1 for $(111)(222) \rightarrow(121221)$ since the first 2 jumps over two variables, then the second 2 jumps over one variable and the last 2 does not jump. However some care has to be taken since the R.H.S. of the second equation of (9) may contain some words which are not of the Roby type. Indeed, it may happen that one of the two subsequences are not of the Roby type. This means in particular that we have to use the rules of reduction of words in order to obtain only elements in the Poincaré-Birkhoff-Witt basis. For instance, if one calculate using (9) $\Delta\left(V_{1212}\right)$ one obtains terms like $V_{122}$ which are not of the Roby type, although for $\Delta\left(V_{11}\right)$ and $\Delta\left(\Delta_{221}\right)$ we only obtain Roby words. Using the rule of reduction, in the first case, one finally obtains

$$
\begin{aligned}
\Delta V_{(11)} & =V_{(11)} \otimes 1+(1+q) V_{1} \otimes V_{1}+1 \otimes V_{(11)} \\
\Delta V_{(12)} & =V_{(12)} \otimes 1+V_{1} \otimes V_{2}+q V_{2} \otimes V_{1}+1 \otimes V_{(12)} \\
\Delta V_{(221)} & =V_{(221)} \otimes 1+V_{22} \otimes V_{1}+\left(q+q^{2}\right) V_{(21)} \otimes V_{2}+\left(q+q^{2}\right) V_{1} \otimes V_{(22)}+V_{2} \otimes V_{(21)}+1 \otimes V_{\left(2^{2} 1\right)} . \\
\Delta\left(V_{1212}\right) & =V_{1212} \otimes 1+\left(V_{1} \otimes V_{212}-q V_{2} \otimes V_{121}-q V_{2} \otimes V_{211}-q^{2} V_{1} \otimes V_{212}-q^{2} V_{1} \otimes V_{221}+V_{2} \otimes V_{121}\right) \\
& +\left(V_{12} \otimes V_{12}+q V_{11} \otimes V_{22}+q^{2} V_{12} \otimes V_{21}+q^{2} V_{21} \otimes V_{12}+V_{22} \otimes V_{11}+q V_{12} \otimes V_{12}\right) \\
& +\left(V_{121} \otimes V_{2}-q V_{211} \otimes V_{1}-q V_{221} \otimes V_{1}-q^{2} V_{121} \otimes V_{2}-q^{2} V_{211} \otimes V_{2}+V_{212} \otimes V_{1}\right)+1 \otimes V_{1212} \\
& -\frac{1}{2}\left(q V_{2} \otimes P_{2}+q^{2} V_{1} \otimes P_{1}+q P_{1} \otimes V_{1}+q^{2} P_{2} \otimes V_{2}\right)
\end{aligned}
$$

\section{DUAL OF THE HOPF ALGEBRA ASSOCIATED TO LIE ALGEBRAS OF ORDER THREE}

Having endowed the universal enveloping algebra with a Hopf algebra structure, in this section we construct its dual. We mainly stress on the algebra structure of $\mathcal{U}(\mathfrak{g})^{*}$ and define a natural coproduct.

\section{A. Associative algebra structure on $\mathcal{U}(\mathfrak{g})^{*}$}

Let $\mathfrak{g}$ be an elementary Lie algebra of order three. We have provided $\mathcal{U}(\mathfrak{g})$ with a Hopf algebra structure, we would like to define now the Hopf dual $\mathcal{U}(\mathfrak{g})^{*}$. In the infinite-dimensional case the notion of dual of a Hopf algebra $H$ is more 
involved. There is in fact two ways to define this notion. In the first approach we restrict to some subset $H^{\circ} \subset H^{*}$ with the appropriate properties. In the second approach we just focus on the pairing (see e.g. [24, 25]). Since we were able to construct a PBW basis of $\mathcal{U}(\mathfrak{g})$, we follow the second approach and we identify the generators of $\mathcal{U}(\mathfrak{g})^{*}$. Let us consider a PBW basis of $\mathcal{U}(\mathfrak{g})$ associated to a basis $\left\{X_{i}, Y_{j}\right\}$ of $\mathfrak{g}$. We have seen that such a basis is written as words $X_{I} Y_{J}$ where $X_{I}$ is a PBW word of $\mathcal{U}(\mathfrak{g})_{0}$ and $Y_{J}$ a Roby word. If $X_{I} Y_{J}$ is an element of the basis of $\mathcal{U}(\mathfrak{g})$, we denote by $\Psi^{I J}$ the corresponding dual element i.e. with the pairing $\Psi^{I J}\left(X_{K} Y_{L}\right)=\delta^{I}{ }_{K} \delta^{J}{ }_{L}$. To simplify the notations we put $\alpha^{I}=\Psi^{I \varnothing}$ (i.e. the dual vector of the word $\left.X_{I}\right), \theta^{J}=\Psi^{\varnothing J}$ (the dual vector of the Roby word $Y_{J}$ ).

Let $\mathcal{U}(\mathfrak{g})^{*}$ be the dual vector space. The following product

$$
M: \mathcal{U}(\mathfrak{g})^{*} \times \mathcal{U}(\mathfrak{g})^{*} \longrightarrow \mathcal{U}(\mathfrak{g})^{*}
$$

given by

$$
M(f, g)(V)=\rho(f, g) \Delta(V)
$$

with

$$
\rho(f, g)(u \otimes v)=f(u) g(v)
$$

defines an unitary associative algebra structure on $\mathcal{U}(\mathfrak{g})^{*}$. In particular we have

$$
1(a)=\epsilon(a)
$$

for every $a$ in $\mathcal{U}(\mathfrak{g})$, where $\epsilon$ is the counit of $\mathcal{U}(\mathfrak{g})$.

Moreover, if in the basis $\left\langle Z_{I J}=X_{I} Y_{J}\right\rangle$ the coproduct writes

$$
\Delta Z_{I J}=\delta_{I J}{ }^{K L M N} Z_{K L} \otimes Z_{M N}
$$

in the dual basis we have

$$
M\left(\Psi^{I J}, \Psi^{K L}\right)=\delta_{M N}{ }^{I J K L} \Psi^{M N}
$$

Proposition 2 As a vector space we have the following isomorphism,

$$
\mathcal{U}(\mathfrak{g})^{*} \cong \mathbb{C}\left[\mathfrak{g}_{0}\right] \otimes \Lambda\left(\mathfrak{g}_{1}, 3\right)
$$

where $\mathbb{C}\left[\mathfrak{g}_{0}\right]$ is the algebra of polynomials in $\operatorname{dim} \mathfrak{g}_{0}$ variables and $\Lambda\left(\mathfrak{g}_{1}, 3\right)$ the three-exterior algebra in dim $\mathfrak{g}_{1}$ variables. Moreover $\left\{\alpha^{i}, \theta^{j}\right\}, i=1, \ldots, \operatorname{dim} \mathfrak{g}_{0}, j=1, \ldots, \operatorname{dim} \mathfrak{g}_{1}$ are the generators of the associative algebra $\mathcal{U}(\mathfrak{g})^{*}$ 
Proof We first prove that the zero-graded part is isomorphic to the set of polynomials. Recall that $X_{\ell \ell}=\frac{X_{\ell}^{2}}{2}$ and for $\ell<s$ we have $X_{\ell s}=X_{\ell} X_{s}$. Since

$$
\Delta\left(X_{\ell} X_{s}\right)=X_{\ell} \otimes X_{s}+X_{s} \otimes X_{\ell}+X_{\ell} X_{s} \otimes 1+1 \otimes X_{\ell} X_{s}
$$

we have

$$
M\left(\alpha^{i_{1}}, \alpha^{i_{2}}\right)= \begin{cases}\alpha^{i_{1} i_{2}} & i_{1}<i_{2}, \\ \alpha^{i_{2} i_{1}} & i_{2}<i_{1}, \\ \alpha^{i_{1} i_{2}} & i_{1}=i_{2} .\end{cases}
$$

Furthermore, by induction we prove that

$$
M\left(\alpha^{i_{1}}, \alpha^{i_{2}}, \ldots, \alpha^{i_{p}}\right)=\alpha^{i_{1} i_{2} \ldots i_{p}}
$$

as soon as $i_{1}<i_{2}<\ldots<i_{p}$. Then the dual vectors $\alpha^{i}, i=1, \ldots, \operatorname{dim} \mathfrak{g}_{0}$ generate the family $\left\{\alpha^{I}\right\}$. It is also easy to prove by induction that the product in $\mathcal{U}\left(\mathfrak{g}_{0}\right)^{*}$ is commutative. Indeed this last property is simply a consequence of the cocommutativity of the coproduct in $\mathcal{U}\left(\mathfrak{g}_{0}\right)$.

We consider now the product in the graded sector. From

$$
\Delta\left(Y_{\ell} Y_{s}\right)=Y_{\ell} \otimes Y_{s}+q Y_{s} \otimes Y_{\ell}+Y_{\ell} Y_{s} \otimes 1+1 \otimes Y_{\ell} Y_{s}
$$

we get

$$
M\left(\theta^{j_{1}}, \theta^{j_{2}}\right)=\theta^{j_{1} j_{2}}+q \theta^{j_{2} j_{1}}
$$

for all $j_{1}, j_{2} \in\left\{1, \ldots, \operatorname{dim} \mathfrak{g}_{1}\right\}$. We deduce that the dual vectors $\theta^{j}$ generate the dual vectors of type $\theta^{j_{1} j_{2}}$. Now we consider a Roby word $Y_{\ell m n}$ of length 3. This implies that $(\ell m n)$ is a Roby sequence (we have not $\ell \leq m \leq n$ ). In this case

$$
\begin{aligned}
\Delta\left(Y_{\ell m n}\right) & =Y_{\ell} Y_{m} Y_{n} \otimes 1+Y_{\ell} Y_{m} \otimes Y_{n}+q Y_{\ell} Y_{n} \otimes Y_{m}+q^{2} Y_{m} Y_{n} \otimes Y_{\ell} \\
& +Y_{\ell} \otimes Y_{m} Y_{n}+q Y_{m} \otimes Y_{\ell} Y_{n}+q^{2} Y_{n} \otimes Y_{\ell} Y_{m}+1 \otimes Y_{\ell} Y_{m} Y_{n}
\end{aligned}
$$

This means in particular that

$$
M\left(\theta^{j}, \theta^{j j}\right)=0 .
$$

For $j_{i}<j_{2}$ we have 


$$
\begin{aligned}
& M\left(\theta^{j_{1}}, \theta^{j_{1} j_{2}}\right)=q^{2} \theta^{j_{1} j_{2} j_{1}} \\
& M\left(\theta^{j_{1}}, \theta^{j_{2} j_{1}}\right)=\theta^{j_{1} j_{2} j_{1}}-\theta^{j_{2} j_{1} j_{1}} \\
& M\left(\theta^{j_{2}}, \theta^{j_{1} j_{1}}\right)=\theta^{j_{2} j_{1} j_{1}}+q \theta^{j_{1} j_{2} j_{1}}
\end{aligned}
$$

and for $j_{1} \neq j_{2} \neq j_{3}$ we obtain

$$
\begin{array}{ll}
M\left(\theta^{j_{1}}, \theta^{j_{2} j_{3}}\right)=\theta^{j_{1} j_{2} j_{3}}+q \theta^{j_{2} j_{1} j_{3}}+q^{2} \theta^{j_{2} j_{3} j_{1}}, & \text { if }\left(j_{1} j_{2} j_{3}\right),\left(j_{2} j_{1} j_{3}\right) \text { and }\left(j_{2} j_{3} j_{1}\right) \text { are Roby sequences, } \\
M\left(\theta^{j_{1}}, \theta^{j_{2} j_{3}}\right)=q \theta^{j_{2} j_{1} j_{3}}+q^{2} \theta^{j_{2} j_{3} j_{1}}, & \text { if }\left(j_{1} j_{2} j_{3}\right) \text { is not a Roby sequence, } \\
M\left(\theta^{j_{1}}, \theta^{j_{2} j_{3}}\right)=\theta^{j_{1} j_{2} j_{3}}+q^{2} \theta^{j_{2} j_{3} j_{1}}, & \text { if }\left(j_{2} j_{1} j_{3}\right) \text { is not a Roby sequence, } \\
M\left(\theta^{j_{1}}, \theta^{j_{2} j_{3}}\right)=\theta^{j_{1} j_{2} j_{3}}+q \theta^{j_{2} j_{1} j_{3}}, & \text { if }\left(j_{2} j_{3} j_{1}\right) \text { is not a Roby sequence. }
\end{array}
$$

These formulæ can be unified as follows we have $M\left(\theta^{\ell}, \theta^{m n}\right)=\theta^{\ell m n}+q \theta^{m \ell m}+q^{2} \theta^{m n \ell}$, but in this last expression we just keep the terms corresponding to Roby sequences. For instance if $(\ell m n)$ is not a Roby sequence we do not have the first term in the sum above. Thus, the dual vectors $\theta^{j}$ generate the vectors $\theta^{\ell m n}$ with $(\ell m n)$ a Roby sequence. We now show that the vectors $\theta^{j}$ generates the three-exterior algebra that is $M\left(\theta^{\ell}, \theta^{m}, \theta^{n}\right)+$ perm. $=0$. We obviously have $M\left(\theta^{j}, \theta^{j}, \theta^{j}\right)=0$. (The multiplication in $\mathcal{U}(\mathfrak{g})^{*}$ is associative since the coproduct in $\mathcal{U}(\mathfrak{g})$ is coassociative.) For $j_{1}<j_{2}$, we have

$$
\begin{aligned}
& M\left(\theta^{j_{1}}, \theta^{j_{1}}, \theta^{j_{2}}\right)=-\theta^{j_{1} j_{2} j_{1}}-q \theta^{j_{2} j_{1} j_{1}}, \\
& M\left(\theta^{j_{1}}, \theta^{j_{2}}, \theta^{j_{1}}\right)=2 \theta^{j_{1} j_{2} j_{1}}-\theta^{j_{2} j_{1} j_{1}}, \\
& M\left(\theta^{j_{2}}, \theta^{j_{1}}, \theta^{j_{1}}\right)=-\theta^{j_{1} j_{2} j_{1}}-q^{2} \theta^{j_{2} j_{1} j_{1}},
\end{aligned}
$$

thus $M\left(\theta^{j_{1}}, \theta^{j_{1}}, \theta^{j_{2}}\right)+$ perm. $=0$. Finally, assume now that $j_{1}<j_{2}<j_{3}$ (this means that $\left(j_{1} j_{3} j_{2}\right),\left(j_{2} j_{3} j_{1}\right),\left(j_{2} j_{1} j_{3}\right),\left(j_{3} j_{1} j_{2}\right)$ and $\left(j_{3} j_{2} j_{1}\right)$ are Roby sequences although $\left(j_{1} j_{2} j_{3}\right)$ is not a Roby sequence), we have

$$
\begin{aligned}
& M\left(\theta^{j_{1}}, \theta^{j_{2}}, \theta^{j_{3}}\right)=q^{2} \theta^{j_{2} j_{3} j_{1}}+q^{2} \theta^{j_{3} j_{1} j_{2}}+q \theta^{j_{1} j_{3} j_{2}}+q \theta^{j_{2} j_{1} j_{3}}+\theta^{j_{3} j_{1} j_{2}} \\
& M\left(\theta^{j_{2}}, \theta^{j_{3}}, \theta^{j_{1}}\right)=\theta^{j_{2} j_{3} j_{1}}+q^{2} \theta^{j_{3} j_{1} j_{2}}+\theta^{j_{1} j_{3} j_{2}}+q \theta^{j_{2} j_{1} j_{3}}+q \theta^{j_{3} j_{2} j_{1}} \\
& M\left(\theta^{j_{3}}, \theta^{j_{1}}, \theta^{j_{2}}\right)=q^{2} \theta^{j_{2} j_{3} j_{1}}+\theta^{j_{3} j_{1} j_{2}}+q \theta^{j_{1} j_{3} j_{2}}+\theta^{j_{2} j_{1} j_{3}}+q \theta^{j_{3} j_{2} j_{1}} \\
& M\left(\theta^{j_{1}}, \theta^{j_{3}}, \theta^{j_{2}}\right)=\theta^{j_{2} j_{3} j_{1}}+q^{j_{3} j_{1} j_{2}}+\theta^{j_{1} j_{3} j_{2}}+q^{2} \theta^{j_{2} j_{1} j_{3}}+q^{2} \theta^{j_{3} j_{2} j_{1}} \\
& M\left(\theta^{j_{2}}, \theta^{j_{1}}, \theta^{j_{3}}\right)=q \theta^{j_{2} j_{3} j_{1}}+\theta^{j_{3} j_{1} j_{2}}+q^{2} \theta^{j_{1} j_{3} j_{2}}+\theta^{j_{2} j_{1} j_{3}}+q^{2} \theta^{j_{3} j_{2} j_{1}} \\
& M\left(\theta^{j_{3}}, \theta^{j_{2}}, \theta^{j_{1}}\right)=q \theta^{j_{2} j_{3} j_{1}}+q \theta^{j_{3} j_{1} j_{2}}+q^{2} \theta^{j_{1} j_{3} j_{2}}+q^{2} \theta^{j_{2} j_{1} j_{3}}+\theta^{j_{3} j_{2} j_{1}}
\end{aligned}
$$

and thus $M\left(\theta^{j_{1}}, \theta^{j_{1}}, \theta^{j_{3}}\right)+$ perm. $=0$.

To end the proof in the graded sector, we observe that the coproduct for Roby words of length greater than three is given by the second equation of (9) eventually corrected by terms similar to those appearing in the last equation of 
(10) (when in the right hand side there is terms which are not of the Roby type). This means that if one calculates $M\left(\theta^{j_{1}}, \theta^{j_{2} \cdots j_{n}}\right)$ two types of terms will be obtained

1. $\theta^{j_{1} j_{2} \cdots j_{n}}+q \theta^{j_{2} j_{1} \cdots j_{n}}+\cdots+q^{n-1} \theta^{j_{2} \cdots j_{n} j_{1}}$, where in the previous summation the non-Roby words are excluded;

2. terms of the type above coming from the reduction in the PBW basis of the non-Roby words which appear in the coproduct (9).

This means that dual vectors $\left\{\theta^{j}\right\}_{j=1, \ldots, \operatorname{dim} \mathfrak{g}_{1}}$ generate the family $\theta^{J}$, and in particular that $<\theta^{J}, J$ Roby sequence $>$ is isomorphic to the three-exterior algebra.

Moreover the non-Roby terms in the coprodruct $\Delta V_{J}$ will induce a product which mixes $\alpha-$ and $\theta$-types of terms. This means that we have $M\left(\alpha^{i}, \theta^{j}\right)=\Psi^{i j}$ plus possibly some terms involving $\theta^{j_{1} j_{2} j_{3} j_{4}}$. Then $\left\{\alpha^{i}, \theta^{j}\right\}, i=1, \ldots, \operatorname{dim} \mathfrak{g}_{0}, j=1, \ldots, \operatorname{dim} \mathfrak{g}_{1}$ are the generators of the associative algebra $\mathcal{U}(\mathfrak{g})^{*}$. It is important to notice that the variables $\alpha^{i}$ and $\theta^{j}$ do not commute. For instance for the algebra $\mathfrak{i s o}_{3}(1,3)$ given in Example 2. looking to $\Delta V_{1212}$ in (10) shows explicitly that $\theta^{1} \alpha^{1} \neq \alpha^{1} \theta^{1}$. Thus as a vector space we have the following isomorphism $\mathcal{U}(\mathfrak{g})^{*} \cong \mathbb{C}\left[\mathfrak{g}_{0}\right] \otimes \Lambda\left(\mathfrak{g}_{1}, 3\right)$. Q.E.D.

\section{Remarks.}

1. In order to respect the grading structure of the algebra, since the elements of $\mathfrak{g}_{1}$ are of grade-one, we assume here that the variables $\theta^{j}$ are of grade two.

2. In [28], the vectorial description of $\mathcal{U}(\mathfrak{g})^{*}$ does not correspond to the result of proposition 3 . In fact, in this paper, the authors do not use Roby algebras to describe the dual of the enveloping algebra.

\section{B. Hopf algebra structure on $\mathcal{U}(\mathfrak{g})^{*}$}

As the algebra $\mathcal{U}(\mathfrak{g})$ is graded, we can put a coalgebra structure on its dual vectorial. To define this coproduct, we consider the vectorial dual basis $\left\{\Psi^{M N}\right\}$ of the PBW basis $\left\{Z_{I J}\right\}$ of $\mathcal{U}(\mathfrak{g})$. If we put

$$
Z_{I J} \cdot Z_{K L}=\mu_{I J K L}{ }^{M N} Z_{M N}
$$

then the linear map

$$
\Delta: \mathcal{U}(\mathfrak{g})^{*} \longrightarrow \mathcal{U}(\mathfrak{g})^{*} \otimes \mathcal{U}(\mathfrak{g})^{*}
$$

given by

$$
\Delta\left(\Psi^{M N}\right)=\mu_{I J K L}{ }^{M N} \Psi^{I J} \otimes \Psi^{K L} .
$$


satisfies in particular

$$
(\Delta f)(X \otimes Y)=f(X Y)
$$

for every $f \in \mathcal{U}(\mathfrak{g})^{*}$ and $X, Y \in \mathcal{U}(\mathfrak{g})$. We deduce

$$
(\Delta \otimes I d) \Delta\left(\Psi^{M N}\right)=\mu_{I J K L}{ }^{M N} \Delta\left(\Psi^{I J}\right) \otimes \Psi^{K L}=\mu_{I J K L}{ }^{M N} \mu_{R S T U}{ }^{I J} \Psi^{R S} \otimes \Psi^{T U} \otimes \Psi^{K L}
$$

and

$$
(I d \otimes \Delta) \Delta\left(\Psi^{M N}\right)=\mu_{I J K L}^{M N} \Psi^{I J} \otimes \Delta\left(\Psi^{K L}\right)=\mu_{I J K L}{ }^{M N} \mu_{R S T U}{ }^{K L} \Psi^{I J} \otimes \Psi^{R S} \otimes \Psi^{T U}
$$

and the coassociativity is given by

$$
\mu_{I J K L}^{M N} \mu_{R S T U}^{I J}=\mu_{I J K L}^{M N} \mu_{R S T U}^{K L} .
$$

This last identity is just a consequence of the associativity of the product in $\mathcal{U}(\mathfrak{g})$. This associativity being itself a consequence of the independence of the way we reduce the non-Roby word in $\mathcal{U}\left(\mathfrak{g}_{1}\right)$.

However, we have seen that the PBW basis of $\mathfrak{g}_{1}$ (noted $B_{1}$ ) is strongly related to the Roby elements $Y_{I}$, or to the Roby sequences $I$. This leads to a difficulty. Its is obvious that the set of Roby sequences is not a representation of $\mathfrak{g}_{0}$. This means that we may obtain results where the $\mathfrak{g}_{0}$-equivariance is not manifest. For instance, if we consider the words of length three of the type $Y_{112}, Y_{121}, Y_{211}$ (i.e. when two indices are equal) only the last two are of the Roby type (since $1 \leq 1 \leq 2$ ). However, if one chooses the cubic elements of $B_{1}$ to be $Y_{j_{1} j_{2} j_{1}}, Y_{j_{2} j_{1} j_{1}}$ with $j_{1}<j_{2}$ and $Y_{j_{1} j_{1} j_{2}}, Y_{j_{1} j_{2} j_{1}}$ when $j_{1}>j_{2}$ we will have a result where the $\mathfrak{g}_{0}$-equivariance will not be manifest. Indeed, it might happen that there is a $G_{0}$ (the Lie group of $\mathfrak{g}_{0}$ ) transformation that maps the Roby words $Y_{121}, Y_{211}$ to $Y_{212}, Y_{122}$. But the last word is not a Roby word. To solve this problem, we take a modified Roby basis for which the $\mathfrak{g}_{0}-$ equivariance would be manifest. Namely, we take the words $Y_{j_{1} j_{1} j_{2}}, Y_{j_{2} j_{1} j_{1}}, j_{1} \neq j_{2}$ to be the modified Roby elements of $B_{1}$. We clearly see that when $j_{1}=2, j_{2}=1$ the word $Y_{122}$ is not a Roby word in the sense originally defined, but is a Roby word in the modified sense. This rule is extended to words of length greater than three.

Example 7 For $\mathcal{U}\left(\mathfrak{i s o}_{3}(1,4)\right)^{*}$ the coproduct is given by

$$
\begin{aligned}
\Delta x^{\nu}= & x^{\nu} \otimes 1+1 \otimes x^{\nu}-f_{\alpha \beta, \gamma}{ }^{\nu} \alpha^{\alpha \beta} \otimes x^{\gamma} \\
& \frac{1}{2} \frac{1}{1-q^{2}} \eta_{\mu \rho} \theta^{\mu} \otimes\left(\theta^{\nu} \theta^{\rho}-q \theta^{\rho} \theta^{\nu}\right)+\frac{1}{2} \frac{1}{1-q^{2}} \eta_{\mu \rho}\left(\theta^{\rho} \theta^{\nu}-q \theta^{\nu} \theta^{\rho}\right) \otimes \theta^{\mu} \\
\Delta \alpha^{\mu \nu}= & \alpha^{\mu \nu} \otimes 1+1 \otimes \alpha^{\mu \nu}+\frac{1}{2} F_{\alpha \beta, \gamma \delta}{ }^{\mu \nu} \alpha^{\alpha \beta} \wedge \alpha^{\gamma \delta} \\
\Delta \theta^{\mu}= & \theta^{\mu} \otimes 1+1 \otimes \theta^{\mu}-f_{\alpha \beta, \gamma}{ }^{\mu} \alpha^{\alpha \beta} \otimes \theta^{\gamma},
\end{aligned}
$$

with $f_{\alpha \beta, \gamma}{ }^{\mu}$ and $F_{\alpha \beta, \gamma \delta}{ }^{\mu \nu}$ the structure constant defined in Example 2 and $x^{\mu}, \alpha^{\mu \nu}, \theta^{\mu}$ the dual vectors of $P_{\mu}, L_{\mu \nu}$ and $V_{\mu}$ respectively. 
Finally the antipode and the counit are easily constructed. Indeed, if for a Hopf algebra $H$ we have $S\left(e_{i}\right)=s_{i}{ }^{j} e_{j}$, the pairing $\mathcal{U}(\mathfrak{g})^{*}$ gives for its dual $S^{*}\left(e^{i}\right)=s_{j}{ }^{i} e^{j}$. The counit of $H^{\star}$ is the unit of $H$. Thus we have:

$$
\begin{aligned}
& S^{*}(1)=1, S^{*}\left(x^{\mu}\right)=-x^{\mu}, S^{*}\left(\omega^{\mu \nu}\right)=-\omega^{\mu \nu}, S^{*}\left(\theta^{\mu}\right)=-\theta^{\mu} \\
& \epsilon^{*}(1)=1, \quad \epsilon^{*}\left(x^{\mu}\right)=0, \quad \epsilon^{*}\left(\omega^{\mu \nu}\right)=0, \quad \epsilon^{*}\left(\theta^{\mu}\right)=0 .
\end{aligned}
$$

Remark 3 Up to now we were considered complex elementary Lie algebras of order three. However, such algebras admit real forms. A real elementary Lie algebra of order three is given by a real Lie algebra $\mathfrak{g}_{0}$ and $\mathfrak{g}_{1}$ a real representation of $\mathfrak{g}_{0}$ which satisfy the axioms of complex elementary Lie algebras of order three. Of course when considering a real form of a complex $\mathfrak{g}$ some structures are lost such that the grading map $\varepsilon$, the coproduct etc. At a first glance it seems that the dual algebra is also lost since its product involves explicitly complex numbers. However, we can forget the multiplication laws given previously and just keep the fact that this algebra is simply given by the set of polynomials and the three-exterior algebra. This is possible since $\Lambda\left(\mathfrak{g}_{1}, 3\right)$ can be defined consistently as a real algebra.

\section{CONCLUSION AND PERSPECTIVE}

In this paper we have studied on formal ground a particular class of ternary algebras named Lie algebras of order three. We have then shown that one is able to associate to Lie algebras of order three a corresponding universal enveloping algebra. A Poincaré-Birkhoff-Witt theorem has been proven in this context. It turns out that the PBW basis is strongly related to the three-exterior algebra (or the Roby algebra). It has then been shown that this universal enveloping algebra can be endowed with a Hopf algebra structure.

At that point one may wonder whether or not we may define groups associated to Lie algebras of order three along the lines one associates Lie groups to Lie algebras. Indeed, if $G$ is a simply connected Lie group, there is a duality between $\mathcal{U}(\mathfrak{g})$ (the universal enveloping algebra of $\mathfrak{g}$, the Lie algebra of $G$ ) and $\mathcal{F}(G)$ (the vector space of complex

valued functions on $G$ ). This means that $\mathcal{F}(G)$ is isomorphic to a subspace of $\mathcal{U}^{*}(\mathfrak{g})$ (the dual of $\left.\mathcal{U}(\mathfrak{g})\right)[24$, 25]. In other words one may wonder if a similar construction applies in the context of Lie algebras of order three. Incidently, partial results have been obtained in this direction: matrix groups involving matrices with elements belonging to the three-exterior algebra were defined in [27]. The question of the relationship of these matrix groups and possible groups associated to Lie algebras of order three is still open.

Acknowledgments J. Lukierski is gratefully acknowledged for discussions and suggestions.

[1] S. Coleman and J. Mandula, Phys. Rev. 159 (1967) 1251.

[2] R. Haag, J. T. Lopuszanski and M. F. Sohnius, Nucl. Phys. B88 (1975) 257. 
[3] I. Bars and M. Gunaydin, J. Math. Phys. 20 (1979) 1977.

[4] I. Bars I and M. Gunaydin, Phys. Rev. D22 (1980) 1403.

[5] L. Vainerman and R. Kerner, J. Math. Phys 37 (1995) 2553.

[6] R. Kerner, Class. and Quantum Grav. 14 (1A) (1997) A203.

[7] A. Borowiec, N. Bazunova and R. Kerner 2004 Lett. Math. Phys. 67 (2004) 195.

[8] M. Rausch de Traubenberg, Clifford algebras, supersymmetry and $\mathbb{Z}_{n}$-symmetries: Applications in field theory, arXiv:hep-th/9802141 (Habilitation Thesis).

[9] V. T. Filipov, Sibirsk. Math. Zh 26 (1985) 126.

[10] A. V. Gnedbaye, C. R. Acad. Sci. Paris Sér. I Math. 321 (1995) 147.

[11] P. W. Michor and A. M. Vinogradov, Rend. Sem. Mat. Univ. Politec. Torino 54 (1996) 373.

[12] N. Goze and E. Remm, On n-ary algebras given by Gerstenhaber's products, arXiv:0803.0553[math.RA].

[13] J. Bagger and N. Lambert, Phys. Rev. D75, (2007) 045020, arXiv:hep-th/0611108.

[14] M. Rausch de Traubenberg and M. J. Slupinski, J. Math. Phys. 41 (2000) 4556, arXiv:hep-th/9904126

[15] M. Rausch de Traubenberg and M. J. Slupinski, J. Math. Phys. 43 (2002), 5145 arXiv:hep-th/0205113.

[16] M. Goze, M. Rausch de Traubenberg and A. Tanasa, J. Math. Phys. 48 (2007) 093507, arXiv:math-ph/0603008.

[17] M. Rausch de Traubenberg and M. Slupinski, Mod. Phys. Lett. A12 (1997) 3051, hep-th/9609203.

[18] N. Mohammedi, G. Moultaka, M. Rausch de Traubenberg, Int. J. Mod. Phys. A19 (2004) 5585, arXiv:hep-th/0305172.

[19] G. Moultaka, M. Rausch de Traubenberg, A. Tanasa, Int. J. Mod. Phys. A20 (2005) 5779, arXiv:hep-th/0411198.

[20] G. Moultaka, M. Rausch de Traubenberg and A. Tanasa, Proceedings of the XIth International Conference Symmetry Methods in Physics, Prague 21-24 June 2004, arXiv:hep-th/0407168.

[21] M. Rausch de Traubenberg, Pr. Inst. Mat. Nats. Akad. Nauk Ukr. Mat. Zastos., 50, Part 1, 2, 3, Natsional. Akad. Nauk Ukraïni, Inst. Mat., Kiev, 2004, pp. 578-585, arXiv:hep-th/0312066

[22] M. Rausch de Traubenberg, Phys. Atom. Nucl. 71 (2008) 1102, arXiv:hep-th/0612204.

[23] R. Campoamor-Stursberg and M. Rausch de Traubenberg, J. Math. Phys. 49 (2008) 063506, arXiv:0801.2630 [hep-th].

[24] V. Chari and A. Pressley, A Guide to Quantum Groups, Cambridge University Press, Cambridge, 1995.

[25] S. Majid, Foundations of Quantum Group Theory, Cambridge University Press, Cambridge, 1995.

[26] N. Roby, Bull. Sc. Math. 94 (1970) 49.

[27] M. Rausch de Traubenberg, J. Phys. Conf. Ser. 128 (2008) 012060, arXiv:0710.5368 [math-ph].

[28] H. Ahmedov, A. Yildiz and Y. Ucan, J. Phys. A 34 (2001) 6413, arXiv:math.RT/0012058

[29] N. Roby, C. R. Acad. Sc. Paris, 268 (1969) A484; Ph. Revoy, C. R. Acad. Sc. Paris 284 (1977) A985; N. Fleury, M. Rausch de Traubenberg, J. Math. Phys. 33 (1992) 3356; N. Fleury and M. Rausch de Traubenberg, Adv. Appl. Cliff. Alg. 4 (1994) 123 . 